\title{
Caracterização da população canina e felina domiciliada do município de Cachoeiras de Macacu, Rio de Janeiro
}

\author{
[Characterization of the canine and feline domiciled population \\ of Cachoeiras de Macacu, Rio de Janeiro]
}

\section{"Artigo Científico/Scientific Article"}

\author{
Adrielle Spinelli Cruz ${ }^{1}$; Elizabeth Cruz Cardoso ${ }^{2,3}$; Flavio Fernando Batista Moutinho ${ }^{4 *}$
}

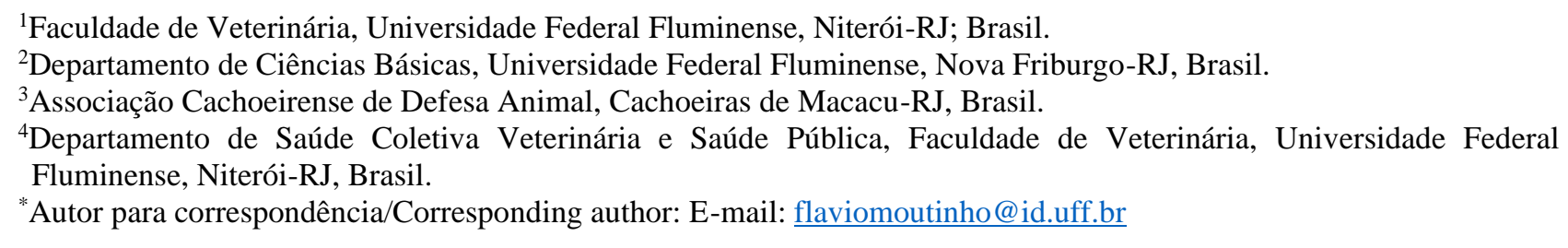

\section{Resumo}

Conhecer o tamanho e o perfil das populações canina e felina é fundamental para a implementação de programas sanitários e de controle da população animal. O presente trabalho teve como objetivo realizar a caracterização da população canina e felina domiciliada do município de Cachoeiras de Macacu, Estado do Rio de Janeiro, Brasil. Trata-se de um estudo transversal descritivo em que foi avaliada uma amostra não probabilística e de conveniência composta por 2.671 domicílios residenciais com levantamento do quantitativo de cães e gatos, machos e fêmeas em cada um dos imóveis. Os dados obtidos foram analisados com técnicas de estatística descritiva (frequência absoluta e relativa de cães e gatos e de machos e fêmeas, razão de cães e gatos por domicílio e razão de cães em relação a gatos). Na amostra estudada foram encontrados 5.082 cães e 1.636 gatos, com razão de 1,9 cães e 0,6 gatos por domicílio, respectivamente. Houve predomínio de imóveis onde havia somente cães $(74,9 \% \%)$, com 3,1 cães para cada gato. Foi detectada, ainda, predominância de machos, tanto em caninos $(60,1 \%)$ quanto em felinos $(54 \%)$. Com base nos resultados se pode concluir que as razões animal/domicilio observadas foram bem mais elevadas do que o esperado, que havia preferência dos tutores por cães em relação a gatos e por machos em relação às fêmeas, tanto em caninos quanto em felinos. Tal resultado pode justificar uma priorização à esterilização de machos em programas de manejo e controle de populações de animais domésticos. Acredita-se que estes resultados possam subsidiar esses programas, bem como àqueles de cunho sanitário, no município de Cachoeiras de Macacu, RJ.

Palavras-chave: cão; gato; perfil populacional.

\begin{abstract}
Knowledge of the size and profile of the canine and feline population is fundamental for the implementation of sanitary and control programs for the animal population. The present work aimed to characterize the canine and feline population living in the city of Cachoeiras de Macacu, Rio de Janeiro, Brazil. This is a transversal descriptive study in which a non-probabilistic and convenience sample composed of 2,671 residential households was surveyed for the number of dogs and cats, males and females in each one of the properties. The data obtained were analyzed using descriptive statistic techniques. In the sample studied were found 5,082 dogs and 1,636 cats, with a ratio of 1.9 and 0.6 per household, respectively. There was a predominance of properties in which there were only dogs, with a 3.10 dogs for each cat, showing owners prefer dogs as domestic animals. A preference was also detected for male compared to female dogs $(60.1 \%)$ and cats $(54 \%)$. Based on the results, it can be concluded that the observed animal/household ratios were much higher than expected, that there was a preference by the owners for dogs instead of cats and males instead of females, both in canines and felines. This result may justify a prioritization of male sterilization in programs of management
\end{abstract}


and control of population of domestic animals. It subsidizes these programs, as well as those of a sanitary nature in the city of Cachoeiras de Macacu, RJ.

Keywords: dog; cat; population profile.

\section{Introdução}

A relação entre os animais domésticos e os humanos é muito antiga. Estudos arqueológicos demonstram que o processo de domesticação do cão ocorreu entre 18.800 e 32.100 atrás (Thalman et al., 2013) e o dos gatos há cerca de cinco ou seis mil anos (Hu et al., 2014). O convívio do ser humano com cães e gatos configura-se como um dos mais estreitos e intensos vínculos entre espécies, e esta relação repercute sobre a saúde das pessoas e dos animais (Vieira et al., 2006).

$\mathrm{O}$ crescimento das populações de animais, como cães e gatos, pode favorecer a transmissão de doenças, especialmente as zoonóticas, já que o processo de domesticação dos animais levou à criação de nichos artificiais que podem se transformar em focos de enfermidades (Forattini, 2004).

Neste contexto, a guarda de animais domésticos, bem como a relação animal / domicílio está associada à forma de ocupação do espaço, ao tipo de domicílio, à atenção dispensada aos animais, bem como a fatores culturais (Canatto et al., 2012). Pelo seu condicionamento à população humana, a população de animais domésticos pode ser afetada pelos mesmos fatores determinantes (FAO, 2003).

Desta forma, estudar as populações de cães e gatos pode gerar informações fundamentais para a realização de intervenções visando o manejo dessas populações, evitando possíveis problemas de saúde pública (ICAMC, 2007). A caracterização dessas populações torna-se a base da estruturação de programas de controle populacional e de zoonoses, minimizando impactos ambientais e sanitários causados pela alta densidade dessas espécies (Canatto et al., 2012). Dentre os principais problemas ocasionados pela alta densidade populacional de animais não domiciliados estão os riscos de mordeduras e transmissão de doenças, a poluição ambiental e os danos às propriedades públicas ou privadas (Reichmann et al., 2000).

Assim, conhecer o tamanho das populações canina e felina é fundamental para obter maior efetividade no planejamento e avaliação dos resultados de ações com objetivo de preservar a saúde de homens e animais (Alves et al., 2005). Nesse contexto, o presente trabalho teve como objetivo caracterizar a população canina e felina domiciliada do município de Cachoeiras de Macacu, Estado do Rio de Janeiro.

\section{Material e Métodos}

O município de Cachoeiras de Macacu integra a Região Metropolitana do Rio de Janeiro, limitando-se com os municípios de Nova Friburgo, Silva Jardim, Rio Bonito, Tanguá, Itaboraí, Guapimirim e Teresópolis (Figura 1). Com área $953,801 \mathrm{~km}^{2}$, possuía população estimada para 2013 de 55.632 habitantes (IBGE, 2014) e Índice de Desenvolvimento Humano Municipal 0,837, que é considerado médio (IBGE, 2010).

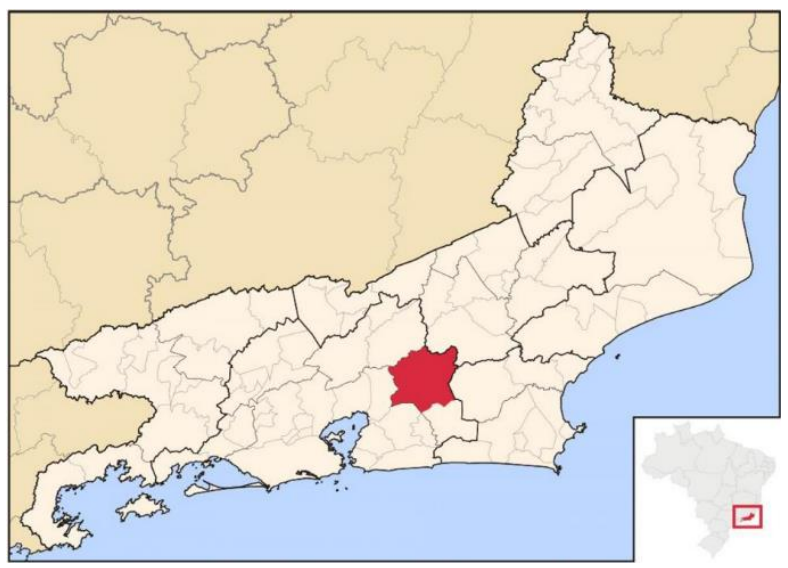

Figura 1. Localização do município de Cachoeiras de Macacu no estado do Rio de Janeiro (Fonte: https://pt.mapof-rio-de-janeiro.com/munic\%C3\%ADpiosmapas/cachoeiras-de-macacu-mapa).

O presente estudo é do tipo transversal descritivo em que foram avaliados dados secundários referentes ao ano de 2013, de uma amostra não probabilística e de conveniência composta por 2.671 domicílios onde residiam cães e gatos do município de Cachoeiras de Macacu.

Os dados levantados foram quantidade de cães e gatos, machos e fêmeas e por domicílio, os quais foram tabulados em planilhas do software Excel $^{\circledR}$ e analisados com técnicas de estatística descritiva (frequência absoluta e relativa de cães e gatos e de machos e fêmeas, razão de cães e gatos por domicílio e razão de cães em relação a gatos). 


\section{Resultados e Discussão}

Nos 2.671 imóveis que compunham a amostra, residiam 6.718 animais, entre cães e gatos, machos e fêmeas, sendo 5.082 cães $(75,6 \%)$ e 1.636 gatos $(24,4 \%)$. A razão encontrada foi de 1,9 cães e 0,6 gatos por domicílio. Canatto et al. (2012), no município de São Paulo, SP, encontraram 1,6 cães por domicílio, que é uma quantidade inferior de cães, mas encontraram 1,69 gatos por domicílio, que é muito superior à quantidade encontrada no presente estudo. De acordo com a Pesquisa Nacional de Saúde de 2013, havia, no Brasil, 1,8 cães por domicílio e 1,9 gatos por domicílio (IBGE, 2015).

Em 74,9\% (n=2001) dos domicílios residiam somente cães, em 18,6\% ( $\mathrm{n}=496)$ cães e gatos simultaneamente e em 6,5\% (n=173) somente gatos. Domingos et al. (2007), em pesquisa realizada em Campo Grande, MS, encontraram proporção menor de domicílios onde existiam somente cães $(64,7 \%)$, semelhante proporção de domicílios onde residiam cães e gatos simultaneamente $(17,7 \%)$ e maior proporção de domicílios onde residiam somente gatos $(24,1 \%)$. Canatto et al. (2012) em São Paulo, SP, encontraram também maior proporção de domicílios onde residiam somente cães, mas seguidos daqueles onde residiam somente gatos e, por fim, daqueles onde residiam ambos.

De acordo com a Pesquisa Nacional de Saúde de 2013, em 44,3\% dos domicílios brasileiros havia ao menos um cão e em $17,7 \%$ ao menos um gato. A população total de cães estimada era de 52,2 milhões e a de gatos 22,1 milhões o que corresponde 2,4 cães para cada gato (IBGE, 2015).

De acordo com o Ministério da Saúde, a meta de cães a serem vacinados contra raiva, em Niterói, em 2013 era de 6.784 (Brasil, 2013). Considerando que essa meta corresponde a $80 \%$ da população total de cães estimada para o município (Brasil, 2009), essa população total estimada de cães seria de 8.480 cães em 2013. Como foram encontrados na presente pesquisa 5.082 cães, isso corresponde a 59,9\% dos cães do município.

Dos 5.082 cães, $60,1 \% \quad(n=3.054)$ eram machos e 39,9\% (n=2.028) fêmeas. Outros estudos também encontraram maior percentual de machos em relação a fêmeas, mas todos com percentual inferior ao encontrado na presente pesquisa, como Caramori Jr. et al. (2003) que encontraram 56,3\% de machos em Cuiabá, MT, Nunes et al. (1997) com 56,2\% de cães machos em Araçatuba, SP,
Domingos et al. (2007) que encontraram 55,7\% em Campo Grande e Cannato et al. (2012) com 52,7\% de machos em São Paulo, SP. Andrade et al. (2008) encontraram proporção menor de cães machos em Araçatuba, SP (49,9\%) em 2004 e, além disso, detectaram grande decrescimento da população de cães machos entre 1999 e 2004 (56,2\% para $49,9 \%$ ).

Do total de 1.636 gatos, $54 \%(\mathrm{n}=884)$ eram machos e $46 \%$ fêmeas $(n=752)$. Tal resultado é próximo ao encontrado por Canatto et al. (2012) em São Paulo, SP, que foi de 52,9\% de machos. Mas o resultado encontrado foi inferior aos encontrados por Domingos et al. (2007) com 62,9\% de machos em Campo Grande, MS e Caramoni Jr. et al. (2003) com 59,3\% de machos em Cuiabá, MT.

O predomínio de machos na população animal pode estar relacionado à preferência dos tutores por esse grupo, evitando inconvenientes ligados ao período de cio, complicações do parto, nascimento e destinação de filhotes, entre outros. (Silva et al., 2010). Pode estar associado, ainda, à questão do uso de cão para guarda, por considerarem os machos como animais menos dóceis e mais valentes. Tal resultado pode justificar uma priorização à esterilização de machos em programas de manejo e controle de populações de animais domésticos.

A razão entre cães e gatos foi de 3,1 cães para cada gato, que foi semelhante à encontrada por Domingos et al. (2007) em Campo Grande, MS, que foi de 3 cães para cada gato e menor que a encontrada por Silva et al. (2010), em Barbacena, MG, de 4,2 cães para cada gato. A relação mais antiga dos humanos com animais domésticos se deu com o cão (Beaver, 2001), o que justifica o predomínio dessa espécie como animal de companhia. Mas é importante salientar que o gato vem crescendo em importância, especialmente por sua fácil adaptação a imóveis de menores dimensões (Beaver, 2005).

\section{Conclusão}

As razões animal/domicilio observadas foram bem mais elevadas do que o esperado, visto ao número total de casas pesquisadas $(\mathrm{n}=2.671)$. $\mathrm{Na}$ amostra estudada houve predomínio de imóveis onde havia somente cães e foram encontrados 3,10 cães para cada gato nos imóveis analisados, mostrando preferência dos tutores por cães em relação a gatos como animais domésticos. Foi detectada nítida preferência dos tutores dos animais 
por machos em relação às fêmeas, tanto em caninos quanto em felinos. Tal resultado pode justificar uma priorização à esterilização de machos em programas de manejo e controle de populações de animais domésticos. Acredita-se que estes resultados possam subsidiar esses programas, bem como àqueles de cunho sanitário, no município de Cachoeiras de Macacu, RJ.

\section{Conflito de interesse}

Os autores declaram não existir conflito de interesse.

\section{Agradecimentos}

À Associação Cachoeirense de Proteção Animal (ACDA) pelo apoio e disponibilização dos dados que propiciaram a realização da presente pesquisa.

\section{Referências}

Alves, M.C.G.P.; Matos, M.R.; Reichmann, M.L.; Dominguez, M.H. Dimensionamento da população de cães e gatos do interior do Estado de São Paulo. Revista de Saúde Pública, 39(6): 891-897, 2005.

Andrade, A.M.; Queiroz, L.H.; Perri, S.H.V.; Nunes, C. M. Estudo descritivo da estrutura populacional canina da área urbana de Araçatuba, São Paulo, Brasil, no período de 1994 a 2004. Cadernos de Saúde Pública, 24(4): 927-932, 2008.

Beaver, B.V. Comportamento canino: um guia para veterinários. São Paulo: Roca, 2001. $431 \mathrm{p}$.

Beaver, B.V. Comportamento felino: um guia para veterinários. São Paulo: Roca, 2005. $384 \mathrm{p}$.

BRASIL. Guia de vigilância epidemiológica. Brasília: Ministério da Saúde, 2009. 816 p.

BRASIL. Ministério da Saúde. Datasus. Programa Nacional de Imunizações. 2013. Disponível em: 〈http://pni.datasus.gov.br>. Acesso em 08 jun. 2017.

Canatto, B.D.; Silva, E. A.; Bernardi, F.; Mendes, M. C. N. C.; Paranhos, N. T.; Dias, R. A. Caracterização demográfica das populações de cães e gatos supervisionados do município de São Paulo. Arquivos Brasileiros de Medicina Veterinária e Zootecnia, 64(6): 1515-1523, 2012.

Caramori Júnior, J.G.; Lubas, M.A.S. Kawatabe, M.S.; Sales, K.G.; Guedes, J.C.; Schmitt, A.C. Inquérito epidemiológico sobre características da população canina e felina de um bairro próximo à zona rural em Cuiabá-MT, visando o controle da raiva animal. Revista da Sociedade Brasileira de Medicina Tropical, 36(3): 419-420, 2003.

Dias, R.A.; Garcia, R.C.; Silva, D.F.; Amaku, M.; Ferreira Neto, J.S.; Ferreira, F. Estimativa de populações canina e felina domiciliadas em zona urbana do Estado de São Paulo. Revista de Saúde Pública, 38(4): 565-570, 2004.

Domingos, I.H.; Rigo, L.; Honer, M.R. Perfil das populações canina e felina no município de Campo Grande, MS. Ensaios e Ciência: Ciências Biológicas, Agrárias e da Saúde, 11(1): 97-103, 2007.

Forattini, O. P. Ecologia, epidemiologia e sociedade. $2^{a}$ ed. São Paulo: Artes Médicas, 2004. $710 \mathrm{p}$.

Hu, Y.; Hu, S.; Wang, W.; Wu, X.; Marshall, F. B.; Chen, X.; Hou, L.; Wang, C. Earliest evidence for commensal processes of cat domestication. Proceedings of the National Academy of Sciences, 111(1): 116-120, 2014.

INSTITUTO BRASILEIRO DE GEOGRAFIA E ESTATÍSTICA - IBGE. Cachoeiras de Macacu. 2010. Disponível em: https://cidades.ibge.gov.br/brasil/rj/cachoeira s-de-macacu/panorama. Acesso em 28 mai. 2018.

INSTITUTO BRASILEIRO DE GEOGRAFIA E ESTATÍSTICA - IBGE. Estimativas populacionais para os municípios brasileiros em 01.07.2014. Disponível em: <https://ww2.ibge.gov.br/home/estatistica/po pulacao/estimativa2014/default.shtm>.

Acesso em: 28 mai. 2018.

INSTITUTO BRASILEIRO DE GEOGRAFIA E ESTATÍSTICA - IBGE. Pesquisa nacional de saúde 2013: acesso e utilização dos serviços de saúde, acidentes e violências. Rio de Janeiro: IBGE; 2015.

INTERNACIONAL COMPANION ANIMAL MANAGEMENT COALITION - ICAMC. Humane dog population management guidance. London: RSPCA; HSI; IFAW; WSAVA; ARC; WSPA, 2007. 22 p.

Nunes, C.M.; Martines, D.A.; Fikaris, S.; Queiroz, L.H. Avaliação da população canina da zona urbana do Município de Araçatuba, São Paulo, SP, Brasil. Revista de Saúde Pública, 31(3): 308-309, 1997.

Reichmann, M.L.A.B.; Figueiredo, A.C.C.; Pinto, H.B.F.; Nunes, V.F.P. Controle de 
populações de animais de estimação. São Paulo: Instituto Pasteur, 2000. 44 p.

Silva, M.H.S.; Silva, J.A.; Magalhães, D.F.; Silva, M.X.; Meneses, J.N.C.; Moreira, E.C. Caracterização demográfica e epidemiológica de cães e gatos domiciliados em Barbacena, MG. Arquivos Brasileiros de Medicina Veterinária e Zootecnia, 62(4): 1002-1006, 2010.

Thalmann, O.; Shapiro, B.; Schuenemann, V. J.; Sawyer, S. K.; Greenfield,D.L.; Sablin, M. V.; López-Giráldez, F.; Domingo-Roura, X.;
Naierala, H.; Uerpmann, H. P.; Laponte, D. M.; Acosta, A. A.; Giemsch, L.; Schmitz, R. W.; Worthington, B.; Buikstra, J. E; Druzhkova, A.; Graphodatsky, A. S.; Ovodov, N. D.; Wahlberg, N.; Freedman, A. H.; Schweizer, R. M.; Koepfli, K.P.; Leonard, J. A.; Meyer, M.; Krause, J.; Pääbo, S.; Green, R. E.; Wayne, R. K. Complete Mitochondrial Genomes of Ancient Canids Suggest a European Origin of Domestic Dogs. Science, 342(6160): 871-874, 2013. 